\title{
Gibt es eine Phänomenologische Netzwerktheorie?
}

\author{
Geschichte, Netzwerk und Identität
}

\author{
Von Jan Arendt Fuhse
}

Zusammenfassung: Der Begriff >Phänomenologische Netzwerktheorie < bezeichnet soziologische Arbeiten aus den letzten 20 Jahren, die sich mit dem Zusammenspiel von symbolischen Formen und der Struktur sozialer Netzwerke beschäftigen und die vor allem von Harrison White und seinen Anhängern stammen. Der Aufsatz analysiert, ob dieser Ansatz die Bedingungen für eine soziologische Theorieschule im Sinne eines >Invisible Colleges` erfüllt. Die Phänomenologische Netzwerktheorie wird damit auf sich selbst angewandt, indem das Zusammenspiel zwischen Sinnformen und der Struktur des Netzwerks ihrer Autoren beschrieben wird. Die folgenden Fragen werden dabei betrachtet: Sind die Autoren des Ansatzes eng miteinander verknüpft? Gibt es einen Kern im Netzwerk oder zerfällt es in Subgruppen? Gibt es eine gemeinsame kollektive Identität? Wie entsteht der Ansatz aus der Kombination von verschiedenen theoretischen Strömungen und in der Auseinandersetzung mit anderen Strömungen in der Soziologie? Diese Fragen werden mit verschiedenen Methoden bearbeitet, die typisch für die Phänomenologische Netzwerkforschung sind: Der Aufsatz beinhaltet eine narrative Beschreibung der Geschichte des Ansatzes (im Kontext anderer soziologischer Theorien), eine formale Analyse des Autorennetzwerks und eine qualitative Analyse von Interviews mit den wichtigsten Autoren.

\section{Einleitung ${ }^{1}$}

Die Netzwerkforschung wird in der Soziologie vor allem als Anwendung eines analytischen Instrumentariums wahrgenommen, der eine dahinter stehende Theorie fehle (Trezzini 1998: 513f). Nach wie vor ist ein Missverhältnis zwischen den ausgefeilten Techniken der Netzwerkanalyse und deren geringer theoretischer Fundierung zu beobachten (Holzer 2006: 107). Demgegenüber nimmt der vorliegende Aufsatz die Arbeiten eines Kreises von Autoren um Harrison White, Charles Tilly, Peter Bearman, Ann Mische, Mustafa Emirbayer und Paul DiMaggio in den Blick, in denen eine eigenständige und innovative Theorie sozialer Netzwerke entwickelt wird. Die Ausgangsthese ist, dass diese Autoren ein Invisible College bilden, dessen Ansatz sich als >Phänomenologische Netzwerktheorie<(PNT) zusammenfassen lässt. Inwiefern die PNT tatsächlich ein Invisible College von miteinander verbundenen $\mathrm{Au}-$ toren mit einem gemeinsamen soziologischen Theorieansatz ist - und ob >Phänomenologische Netzwerktheorie< hierfür das richtige Label ist - wird im Folgenden diskutiert.

Dafür werden die Geschichte, das Netzwerk und die Identität der PNT behandelt. Im Sinne von Harrison White besteht die soziale Welt aus Netzwerken von Sozialbeziehungen, die sinnhaft strukturiert sind. Dem folgend soll die PNT als Netzwerksegment im soziologischen Diskurs betrachtet werden, das durch eine bestimmte Phänomenologie von Identitäten und soziologisch-theoretischen Aussagen zusammengehalten wird und sich dadurch von anderen Netzwerksegmenten unterscheidet. Die zugrunde liegende theoretische Perspektive wird aus den betrachteten Arbeiten übernommen. Es folgt also eine Anwendung der PNT auf sich selbst.

1) Die präsentierte Forschung wurde im Rahmen eines einmonatigen Aufenthalts am Institute for Social and Economic Research and Policy der Columbia University im Herbst 2005 durchgeführt. Ich danke Peter Bearman, Eiko Ikegami, Charles Tilly und Harrison White für ausführliche Hintergrundgespräche sowie allen anderen Teilnehmern der von mir durchgeführten Befragung. Alexander Jäger, John Levi Martin, Lena May, Jochen Mayerl, Sophie Mützel, Marlen Schulz und die anonymen Gutachter haben mit wertvollen Hinweisen und Kritik zu dem Artikel beigetragen.

Soziale Welt 59 (2008), S. $31-52$ 
Zunächst werden die Kriterien für ein solches >Invisible College < im Sinne der PNT skizziert (1). Anschließend wird die Geschichte der PNT als Verbindung von netzwerkanalytischem und phänomenologischem Gedankengut narrativ dargestellt (2). Besonderes Augenmerk liegt dabei auf der Auseinandersetzung mit der Rational Choice-Theorie (3). Es folgt eine Analyse des Netzwerks von Danksagungen und Ko-Autorschaften zwischen den Autoren der PNT. Im ersten Schritt wird untersucht, inwiefern dieses Netzwerk eine Verdichtung im akademischen Diskurs darstellt (4), und im zweiten Schritt, ob sich das Netzwerk der PNT um ein gemeinsames Zentrum organisiert oder ob es in mehrere Subgruppen zerfällt (5). Im sechsten Abschnitt wird die Frage der >Identität $<$ der PNT diskutiert (6).

\section{Invisible Colleges}

Nach Thomas Kuhn sind wissenschaftliche Paradigmen in Gruppen von Wissenschaftlern verankert, die sich gemeinsam an einem Paradigma orientieren und sich dadurch von anderen Gruppen unterscheiden (Kuhn 1970: 180). Wenig beleuchtet wird bei Kuhn die Rolle der internen Kommunikationsstrukturen innerhalb solcher Paradigma-Gruppen. Diana Crane und Nicholas Mullins charakterisieren wenig später diese als >Invisible Colleges< und lenken damit das Augenmerk auf die Netzwerkstrukturen in der Wissenschaft (Crane 1972; Mullins 1973). Solche Invisible Colleges sind wichtige Triebfedern in der modernen Wissenschaft, vor allem dadurch, dass hier neue Paradigmen entwickelt und elaboriert werden (Griffith / Mullins 1972).

Im Sinne der PNT bringen engmaschige Netzwerke in Invisible Colleges eigene Denkrichtungen hervor. Kulturelle Muster und Bedeutungen entstehen in Netzwerken. Andererseits integrieren solche Denkrichtungen die Netzwerke von Invisible Colleges sinnhaft. Harrison White bezeichnet die Gesamtheit der Sinnmuster in einem Netzwerk als >Domäne< (White 1995: 708ff; Mische / White 1998: 702ff). Netzwerke - seien es Invisible Colleges oder Straßengangs - zeichnen sich durch eine bestimmte >Kultur< oder Domäne aus, je nachdem welche Sinnmuster in ihnen verbreitet werden und welche nicht. Das erste Kriterium für die Existenz eines Invisible Colleges ist damit eine gemeinsame theoretische Ausrichtung einer Gruppe von Autoren:

\section{Die betrachteten Autoren zeichnen sich durch gemeinsame theoretische Annahmen aus und unterscheiden sich darin deutlich von anderen Netzwerkpopulationen.}

Diese erste These kann hier nicht ausführlich diskutiert werden. Dafür müsste eine umfangreiche Literaturanalyse durchgeführt werden (inklusive einer formalen Kategorisierung von theoretischen Annahmen). Statt einer solchen Literaturanalyse soll das Augenmerk in diesem Aufsatz auf die Entstehung, das Netzwerk und die Identität der PNT gelenkt werden. Im Folgenden will ich aber kurz einige der Grundgedanken der Phänomenologischen Netzwerktheorie skizzieren:

Die allgemeine Annahme der PNT ist, dass in den Transaktionsprozessen in Netzwerken Sinnformen wie zum Beispiel Wertorientierungen und Einstellungen, kategoriale Grenzziehungen oder Rollenmuster entstehen, die im Umkehrschluss strukturierend auf die Transaktionen in Netzwerken wirken (Erickson 1988; Gould 1995; Lizardo 2006). Genau genommen betont die PNT, dass soziale Netzwerke nur als Sinnstrukturen denkbar sind:

»social networks are seen not merely as locations for, or conduits of, cultural formations, but rather as composed of culturally constituted processes of communicative interaction.« (Mische 2003: 258)

Kultur und Netzwerkstruktur sind demnach eng miteinander verknüpft und nur analytisch voneinander zu trennen. Netzwerke lassen sich als Muster von sinnhaft strukturierten Sozialbeziehungen zwischen Akteuren charakterisieren - sie sind nach Harrison White »phenome- 
nological realities« (White 1992: 65). Netzwerke werden durch relationale Narrative ( >stories $<$ ) zusammen gehalten, die im Austausch zwischen Akteuren entstehen, deren Beziehungen untereinander kennzeichnen und damit nicht zuletzt auch die Identität der beteiligten Akteure selbst definieren (White 1992; Somers 1994; Tilly 2002). Das Netzwerk selbst bringt nicht nur Sinnformen (wie Symbole, Kategorien und Einstellungen) hervor. Sondern ein Netzwerk besteht selbst aus einer Struktur von Erwartungen zwischen Identitäten, die beide erst im Netzwerk ausgehandelt werden.

In diesem Sinne haben Netzwerkforscher den Zusammenhang zwischen Netzwerken und Sinnformen in Kommunen (Martin 2002), Kunstströmungen (White 1993) und sozialen Schichten (Erickson 1996; Lizardo 2006), in sozialen Bewegungen (Gould 1995; Ansell 1997; Mische / Pattison 2000) und eben auch in Invisible Colleges in der Wissenschaft untersucht (Mullins 1973; Collins 1998). Übertragen auf die Wissenschaftslandschaft bedeutet dieser Ansatz, dass etwa Rational Choice, Systemtheorie und Netzwerkforschung in unterschiedlichen Netzwerkkontexten entstanden sind - dass aber andererseits diese unterschiedlichen Forschungsorientierungen die Netzwerkkontexte tendenziell getrennt voneinander halten.

Dieses Wechselspiel von Sinn- und Strukturebene in kollektiven Identitätsphänomenen deckt natürlich nur einen Teil der PNT ab. Viele wichtige Theoriekomponenten können hier nur kurz angeschnitten werden. Außerdem zeichnen sich die hier als >Phänomenologische Netzwerktheorie< zusammengefassten Arbeiten weniger durch ein geschlossenes Theoriegebäude aus als durch eine lose Anlehnung an gemeinsamen Grundgedanken und Konzepten. Das Wichtigste von ihnen ist mit dem Begriff der >strukturellen Äquivalenzく verbunden. >Strukturell äquivalent< werden nach Harrison White solche Positionen genannt, die mit anderen Positionen in ähnlicher Weise verknüpft sind - ohne dabei notwendigerweise miteinander in Kontakt zu stehen (White et al. 1976). Beispiele dafür wären etwa Patrone und Klienten oder Verwandtschaftskategorien (Onkel, Neffen). Solche Rollenkategorien sind wesentlicher Bestandteil der Identität von Akteuren und bestimmen deren Beziehungen untereinander (Mohr 1994: 333f). Dabei wird theoretisch angenommen, dass soziale Kategorien Netzwerkstrukturen ordnen, indem sie Erwartungen für die Beziehungen zwischen Akteuren in Anschlag bringen. Über die Untersuchung von Netzwerken können dann Kategorisierungen von sozialen Rollen rekonstruiert werden. Insofern geht es auch hier um das Zusammenspiel von Netzwerkstrukturen und Sinnmustern (den Rollenkategorien).

Diese wenigen Bemerkungen müssen hier zunächst genügen, um die Forschungsperspektive der PNT zu umreißen. Die folgenden Thesen (2 bis 6) werden dagegen jeweils in einem dazugehörigen Abschnitt (wiederum 2 bis 6) behandelt. Zunächst soll die Geschichte der PNT in den Blick genommen werden. Wie kann es der PNT zufolge zur Entstehung einer soziologischen Theorie kommen? Im Sinne der PNT ist das intellektuelle Feld in Netzwerken strukturiert (Collins 1998; Fuchs 2001; Abbott 2001: 136ff). Bestimmte Denkrichtungen sind dabei in Netzwerkclustern verankert, die miteinander um Aufmerksamkeit konkurrieren. Besonders augenfällig ist dies bei den Sozialwissenschaften, weil diese durch Konflikte zwischen sich widersprechenden Paradigmen geprägt sind (Abbott 2001).

Intellektuelle Kreativität kann dabei auf zwei Arten entstehen (Collins 1998: 131ff): Erstens kann ein bestimmtes Paradigma elaboriert werden, d.h., die Implikationen von bestimmten Grundannahmen oder methodischem Vorgehen werden immer weiter ausgearbeitet. Zweitens gibt es die Möglichkeit der Synthese verschiedener Denkrichtungen, in der Grundannahmen oder Vorgehensweisen kreativ miteinander verknüpft werden. Auf diese Weise entsteht nach Harrison White ein neuer Stil in der Verbindung von zwei alten Stilen (White 1993b). Ein Beispiel ist die Entstehung des Rock'n'Roll in den 1950ern aus der Verbindung von afro-amerikanischen Musikrichtungen wie Blues und Jazz und >weißen< Stilen 
wie Country und Disc-Jockey-Musik (White 1993b: 82ff). Angewandt auf die hier betrachtete Fragestellung ergibt sich die These:

\section{Die PNT entsteht aus der Verknüpfung von vormals getrennten Denkrichtungen.}

Eine besondere Rolle spielt dabei die Auseinandersetzung mit anderen soziologischen Schulen. Die soziologische Theorie ist (genau wie etwa die Philosophie) ein intellektuelles Feld, das sich um Oppositionen zwischen wenigen zentralen Positionen herum strukturiert (Collins 1998: 81f). Die Dynamik des Feldes wird dabei vor allem vom Wechselspiel zwischen diesen zentralen Positionen bestimmt: Theoretische Schulen grenzen sich voneinander ab, starke Schulen splitten sich eventuell auf, schwache Schulen verschwinden oder verbünden sich im Kampf um Aufmerksamkeit gegen die stärkeren Schulen. Der Konflikt zwischen solchen Schulen sorgt dabei nicht nur für eine verstärkte Kohäsion im Inneren. Er sorgt auch dafür, dass intellektuelle Positionen überhaupt als unterschiedlich und eigenständig deutlich werden. Genau diese Logik von Identität und Differenz macht eine soziologische Theorieschule zu einem symbolisch und strukturell von anderen Theorieschulen abgegrenzten sozialen Gebilde. Die dritte zu betrachtende These lautet demnach:

\section{Eine besondere Rolle bei der Genese und Stabilisierung der PNT spielt der Konflikt mit anderen soziologischen Schulen.}

Die Thesen 2 und 3 betreffen die Geschichte der PNT, die in den Abschnitten 2 und 3 narrativ nacherzählt wird. Andererseits ist >Geschichte< (story) aber auch Fachausdruck innerhalb der PNT und steht für die narrative Konstruktion von Identität und Differenz in Sozialbeziehungen (White 1992: 65ff; Somers 1994; Tilly 2002: 8ff, 26ff). So konstruieren soziologische Schulen (wie auch Gangs, Parteien oder ähnliche kollektive Identitätsphänomene) in ihren wechselseitigen Abgrenzungsprozessen die >story< ihres Verhältnisses zueinander - und alle symbolischen Konstrukte und soziale Gebilde bestehen der PNT zufolge nur in den relationalen >stories<, mit denen ihre Verhältnisse zu anderen symbolischen Konstrukten oder sozialen Gebilde >erzählt< werden (s.o.). Diese >Geschichten< von Sozialbeziehungen machen ein Netzwerk aus - in diesem Fall die Landschaft der soziologischen Schulen. Narrative Analysen können dabei einerseits solche relationalen >stories< rekonstruieren. Andererseits sind sie selbst >stories<, mit denen symbolische Konstrukte zueinander in Bezug gesetzt werden. Auf diese Weise spinnt sich Wissenschaft in der beständigen Narration sich aufeinander beziehender $>$ stories $<$ fort.

Nach der Geschichte der PNT wird das Netzwerk ihrer Autoren betrachtet: Wie sieht ein Netzwerk eines Invisible Colleges (oder auch von anderen kollektiven Identitätsphänomenen wie sozialen Bewegungen, Gangs etc.) konkret aus? Zunächst muss ein solches Invisible College eine Verdichtung im allgemeinen Netzwerk der Wissenschaftslandschaft darstellen. Die Autoren eines Invisible Colleges oder auch einer soziologischen Schule allgemein müssen in ihrer Kommunikation eine höhere Dichte aufweisen als nach außen zu benachbarten Schulen. Randall Collins stellt dabei die Bedeutung direkter persönlicher Beziehungen in Invisible Colleges heraus (Collins 1998: 71ff). In persönlichen Beziehungen lassen sich Denkweisen besser austauschen und aushandeln als etwa in dem eher >unpersönlichen< Einfluss über Publikationen. Die vierte These lautet damit:

\section{Es gibt ein dichtes Netzwerk persönlicher Beziehungen von Autoren, die der PNT zuzurechnen sind.}

Allerdings ist es nicht möglich, eine solche >Verdichtung < im akademischen Diskurs ohne erheblichen methodischen Aufwand nachzuweisen. Deswegen soll es mir in erster Linie darum gehen aufzuzeigen, dass die Autoren der PNT (die ich in einer subjektiven Auswahl als 
solche identifiziere) in engem persönlichen Austausch miteinander stehen. Die Verbindungen nach außen - zu anderen soziologischen Schulen oder zu eher methodisch orientierten Netzwerkforschern - können hier nicht weiter in den Blick genommen werden.

Eine genauere Untersuchung des Netzwerks ist allerdings hinsichtlich eines zweiten Kriteriums möglich. Dabei geht es um die Frage, ob das Wissenschaftlernetzwerk tatsächlich eine kohärente Gruppe bildet, oder ob es in mehrere Subgruppen oder Fraktionen zerfällt. Nur im Ausnahmefall sind Gruppen perfekt integriert und weisen Verbindungen zwischen allen Beteiligten auf. Aber wenn es einen gemeinsamen sinnhaften Fokus gibt - eine Identität als Kollektiv -, dann sind die Sozialbeziehungen auf einen gemeinsamen Kern hin orientiert. Dies entspricht dem Modell einer >Involution< nach Harrison White und Stephan Fuchs (White 1992: 35, 75; Fuchs 2001: 51, 191f, 216f). In einer solchen Involution trennt sich ein Netzwerksegment symbolisch und strukturell von umgebenden Sozialbeziehungen ab:

»A working network accomplishes a measure of self-similarity as it focuses attention on itself, distinguishes its identity from other networks and from its own components, and draws boundaries around itself. Much as selves, a self-similar network has an identity, for itself and for others.« (Fuchs 2001: 272)

Um dem Modell eines involuierten Netzwerks zu entsprechen, müssen die Netzwerkverbindungen im Inneren stark verdichtet sein und nach außen »ausfransen« (Fuchs 2001: 281ff). Es ergibt sich eine Struktur von einem Kern, um den sich das Netzwerk kristallisiert, und einer Peripherie, in dem sich Einflüsse mit anderen Netzwerken überschneiden. Die fünfte These zielt auf diese interne Differenzierung des Netzwerks:

\section{Das Netzwerk der PNT gruppiert sich um einen gemeinsamen Fokus und ist deswe- gen intern in Zentrum und Peripherie und nicht in verschiedene Subgruppen unter- teilt.}

Dieser gemeinsame Fokus der PNT ist aber auf der Ebene der Sinnformen zu suchen. So wurde eingangs die gemeinsame Orientierung an einer partikularen Forschungsperspektive in einem Invisible College herausgestellt (These 1). Zu der kulturellen >Domäne< eines kollektiven Identitätsphänomens gehört auch die Orientierung an einer gemeinsamen Identität, die im darüber liegenden Netzwerk der Wissenschaftslandschaft als Zurechnungspunkt für Kommunikation fungiert. Systemtheorie, Rational Choice oder der Symbolische Interaktionismus werden erst durch eine solche Identitätskonstruktion zu Theorieschulen bzw. zu >Akteuren< im Wissenschaftsdiskurs: In einem Netzwerk (wie der soziologischen Wissenslandschaft oder anderen Märkten) kommen verschiedene Netzwerkpopulationen in Kontakt miteinander, konkurrieren um knappe Ressourcen (etwa: Reputation, Universitätsstellen, Forschungsmittel) und ringen deswegen miteinander um die Kontrolle im übergreifenden Netzwerk (White 1992). In dieser Konkurrenz entsteht eine >pecking order<, und zu dieser >Hackordnung \& gehört die Konstruktion von kollektiven und individuellen Identitäten. Das >Labelling ist selbst Bestandteil des Kampfes um die Deutungsmacht im Netzwerk. So wird die Frage nach der Wahrheit in der Wissenschaft zu einer Frage um Loyalitäten und Anhängerschaft, um Netzwerkbande und dem Zugang zu Publikationsorten, Universitätsstellen und Forschungsmitteln. Eine (begrenzte) Analogie dazu sind soziale Bewegungen, deren Deutungsmacht auf den Ansprüchen aufbaut: »We are worthy, united, numerous, and committed.«(Tilly 2002: 120)

Wie oben bereits angesprochen wurde, entstehen und verhärten sich solche kollektiven Identitäten gerade im Widerstreit mit anderen Kollektiven (These 3). Im Sinne von Andrew Abbott gilt: Eine soziologische Theorieschule besteht erst in der Konstruktion von Identität und Differenz (Abbott 1995). Die sechste These betrifft diese Orientierung an einer gemeinsamen Identität: 


\section{Die Kommunikation orientiert sich sowohl innerhalb als auch außerhalb der betrachteten Netzwerkpopulation an einer kollektiven Identität, an einem Label für die Netzwerkpopulation.}

Wie bereits angedeutet, sollen die Thesen 2 bis 6 im Folgenden jeweils in einem eigenen Abschnitt betrachtet werden. Die Frage der gemeinsamen theoretischen Annahmen (These 1) kann wie gesagt nicht genauer untersucht werden, wurde aber in diesem ersten Abschnitt angerissen.

\section{Der >Cultural Turn< in der Netzwerkforschung}

Die Phänomenologische Netzwerktheorie entsteht im Sinne der zweiten These aus der kreativen Verbindung zweier disparater Stile in der Soziologie. Die Grundlage dafür bildet die Netzwerkanalyse, die soziale Phänomene auf der Ebene der Struktur sozialer Beziehungen erklären will. Diese hat sich aus den Anfängen in der soziometrischen und der sozialanthropologischen Forschung heraus bis in die 80er Jahre in den USA zu einem engmaschigen und eigenständigen Netzwerk entwickelt (Scott 2000; 7ff; Freeman 2004: 129ff). Zunehmend werden dabei auch die theoretischen Implikationen der Netzwerkanalyse thematisiert (Wellman 1983; Emirbayer 1997; Beckert 2005; Holzer 2006: 74ff). Im Mittelpunkt dieses Netzwerkes steht schon früh Harrison White, der in den 70er Jahren mit Kollegen die Blockmodell-Analyse entwickelte und an den Universitäten Harvard, Chicago und Columbia Doktoranden wie Mark Granovetter, Paul DiMaggio, Peter Bearman oder Barry Wellman prägte (Mullins 1973: 250ff; Freeman 2004: 121ff). Wie im nächsten Abschnitt zu sehen sein wird, stehen fast alle wichtigen Netzwerkanalytiker und -theoretiker in den USA in direkter Verbindung zu White - die meisten von ihnen als ehemalige Studenten.

Anfang der 90er Jahre wendet sich Harrison White stärker der Verankerung von kulturellen Formen wie Narrativen und Identitäten in sozialen Netzwerken zu (White 1992; 1993). Es entsteht eine Verbindung der Netzwerkforschung mit verschiedenen Forschungssträngen, die allesamt dem >Cultural Turn< in den Sozialwissenschaften zuzurechnen sind: die Analyse von Narrativen, Identität und Frames, die neue Kultursoziologie, die Konversationsanalyse und die Wissenschaftssoziologie. Auf diese Weise werden die Phänomenologie von Sinnformen und die Netzwerkanalyse sozialer Strukturen miteinander verknüpft (Emirbayer / Goodwin 1994; Somers 1994; DiMaggio 1997; Collins 1998; McLean 1998; Fuchs 2001; Gibson 2005). Die >Phänomenologie< der PNT bezieht sich nicht direkt auf die Arbeiten von Edmund Husserl, Alfred Schütz und Thomas Luckmann. Vielmehr fungiert >Phänomenologie< als Sammelbegriff für die verschiedenen Ansätze des >Cultural Turn< der amerikanischen Sozialwissenschaften (Breiger 1995: 117). Zugleich bleiben die genannten Arbeiten in ihren theoretischen Begriffen und Aussagen in gewissem Maße heterogen, wie später noch genauer diskutiert wird. Dies liegt wohl wesentlich daran, dass im amerikanischen Kontext eine engere Verbindung aus Theorie und Empirie gepflegt wird - und dass die theoretische Strukturierung von empirischen Analysen meist Vorrang hat vor der reinen Begriffsarbeit. Dies sorgt aber umgekehrt dafür, dass der theoretische Begriffsapparat der PNT stärker als etwa der der Systemtheorie von empirischen Ergebnissen beeinflusst wird.

Interessanterweise wird die Verknüpfung von Netzwerkforschung und Kultursoziologie unabhängig voneinander von Autoren aus verschiedenen Richtungen gefordert (Fine / Kleinman 1983; Hannerz 1992; Brint 1992; DiMaggio 1992). Offensichtlich legte die damalige Konfiguration von Netzwerken in der Soziologie eine solche Verbindung nahe. In dieser Bewegung kommt Harrison White eine besondere Bedeutung zu. White war zum Zeitpunkt seines >Cultural Turn< bereits zentraler Bezugspunkt des Netzwerks von Netzwerkanalytikern. Sein theoretisches Hauptwerk Identity and Control von 1992 bleibt zwar für den Außenstehenden vor allem beim ersten Lesen schwer verständlich und wäre allein kaum in der Lage, 
eine neue Theorieschule zu begründen. ${ }^{2}$ Aber White hat in seinen Seminaren und Workshops und in der Betreuung von Doktoranden viele wichtige Soziologen in den USA geprägt (Freeman 2004: 125ff). Dass die Innovation der Verbindung aus Kultursoziologie und Netzwerkforschung vor allem ihm zugeschrieben wird, hängt stark mit seiner Position im Netzwerk zusammen. Ähnliches hat Randall Collins für Philosophen wie Plato, Konfuzius und Hegel nachgewiesen: Auch diese wurden erst im Netzwerk ihrer Schüler zu den eminenten Figuren gemacht, als die wir sie heute kennen (Collins 1998). Eine Identität von Personen entsteht erst im Netzwerk von Sozialbeziehungen - das Netzwerk ist wichtiger als das Individuum.

Diese Bemerkungen sollen die intellektuelle Leistung von Harrison White nicht mindern. Eher geht es darum, die Bedingungen für die Entstehung einer intellektuellen Innovation und einer soziologisch-theoretischen Schule zu rekonstruieren - und wie dies konkret bei der PNT aussieht. Die PNT entsteht zu Beginn der 90er Jahre aus dem selbstbewussten und fest etablierten Netzwerk der Netzwerkanalytiker heraus, indem Anregungen aus der neueren Kultursoziologie (in ihren verschiedenen Formen) übernommen werden und sowohl theoretisch wie methodisch in das Repertoire der Netzwerkanalyse Eingang finden. Nicht alle Netzwerkforscher machen diesen Schritt mit - etwa Barry Wellman, Ronald Burt oder Mark Granovetter bleiben bei einer eher strukturalistischen Version der Netzwerkforschung ohne Bezug auf kulturelle Formen. Auch zwischen den hier der PNT zugeordneten Autoren findet sich unterschiedlich stark ausgeprägtes Interesse für Sinnformen - und teilweise auch leicht unterschiedliche Vorstellungen über deren Zusammenhang mit der Netzwerkstruktur.

Das neue Netzwerk der PNT entsteht nicht so sehr durch eine Verbindung von alten Netzwerkclustern, sondern durch eine Aufsplittung des Netzwerkes der Netzwerkforscher. Innerhalb der Netzwerkforschung übernimmt ein Netzwerksegment einen stärkeren Fokus auf kulturelle Formen - es kommt im Sinne von Andrew Abbott zur kreativen Kreuzung von Leitgegensätzen (Abbott 2001: 10ff). Erhalten bleibt dabei der starke Bezug zur empirischen Forschung und zu komplexen mathematischen Modellierungen. Neu ist das Interesse an symbolischen Formen und deren Wechselwirkung mit der Struktur sozialer Netzwerke. Diese neue Betonung der Phänomenologie von sozialen Netzwerken bekommt im intellektuellen Feld starke Aufmerksamkeit, weil sie Stile aus verschiedenen Schulen miteinander verbindet.

\section{Auseinandersetzung mit Rational Choice}

Ein wichtiger Grund für diese Aufmerksamkeit für die Phänomenologische Netzwerktheorie liegt im Konflikt mit der Rational Choice-Theorie und damit in der Struktur des soziologisch-theoretischen Feldes in den USA in den 90er Jahren. Dies entspricht der dritten These, dass der Konflikt mit anderen soziologischen Schulen eine wichtige Rolle bei der Genese und Stabilisierung der PNT spielt.

Die Rational Choice-Theorie nimmt in den 80er und 90er Jahren einen zentralen Platz in der Wissenschaftslandschaft der soziologischen Theorien ein. Verstehende Theorien wie die Ethnomethodologie oder der Symbolische Interaktionismus, die sich weitgehend auf die Beschreibung von Mikro-Phänomenen beschränken, verlieren zu diesem Zeitpunkt an Gewicht (Alexander et al. 1987). Rational Choice tritt dagegen selbstbewusst mit dem Anspruch einer Gesamttheorie des Sozialen mit der Erklärung von Makro-Phänomenen aus einer MikroTheorie des rationalen Handelns und dem Bezug zum methodischen Individualismus der em-

2) Voraussichtlich im Mai 2008 erscheint bei Princeton University Press eine erheblich überarbeitete und erweiterte Zweite Auflage von Identity and Control. 
pirischen Sozialforschung auf und drängt damit in den Mittelpunkt der Aufmerksamkeit der amerikanischen soziologischen Theorie.

Wo eine Theorieströmung den zentralen Aufmerksamkeitsfokus einnimmt, bieten sich strukturelle Möglichkeiten für Aufmerksamkeit durch den Konflikt mit ihr (Collins 1998: 80f, 379ff). Dafür müssen sich andere Theorierichtungen verbünden und ihre Kräfte bündeln. Die Netzwerkanalyse mit ihrer methodischen Ausrichtung auf zwischenmenschliche Strukturen und die Verstehende Soziologie (Ethnomethodologie, Symbolischer Interaktionismus, Kultursoziologie) mit ihrem Blick auf Sinnstrukturen lehnen die Reduktion des Menschen auf einen kühl kalkulierenden homo oeconomicus gleichermaßen ab (auch wenn dieses Bild des homo oeconomicus inzwischen erheblich erweitert wurde). Insofern bietet sich eine Verbindung dieser beiden Gegenpositionen an, um der Rational Choice-Theorie mit vereinten Kräften entgegen zu treten. So lässt sich wohl erklären, warum in den 80ern und zu Beginn der 90er Jahre verschiedene Autoren aus unterschiedlichen Richtungen teilweise unabhängig voneinander eine Verbindung von Verstehender Soziologie und Netzwerkanalyse forderten (s.o.).

Die PNT argumentiert, dass soziale Strukturen im Eigensinn von sozialen Transaktionen zwischen Akteuren entstehen und reproduziert werden - und dass selbst Identität und Orientierungen von Akteuren Ergebnisse dieser überpersönlichen Prozesse sind (Leifer 1991; White 1992; Somers 1994; Emirbayer 1997; Emirbayer / Mische 1998; Tilly 2002). Dieses theoretische Argument gegen den methodologischen Individualismus verbindet Motive aus Strukturtheorien, aus Symbolischem Interaktionismus und Konstruktivismus und aus der Neueren Kultursoziologie mit ihrem Fokus auf Narrative und Identitäten. Insofern zieht die Phänomenologische Netzwerktheorie Theoriefiguren unterschiedlicher Provenienz zusammen und verbindet sie zu einer kraftvollen Gegenposition zu Rational Choice. Die PNT bietet eine Synthese der Theoriestränge an, die gleichermaßen gegen methodologischen Individualismus und reine Variablensoziologie stehen (Abbott 1997), und die mit dem Siegeszug von Rational Choice in die Defensive geraten sind.

Diese Auseinandersetzung mit Rational Choice prägt die >story< der Netzwerktheorie. Whites Schüler Barry Wellman propagierte in den 80ern erstmals eine Theorie sozialer Netzwerke über die reine Netzwerkanalyse hinaus (Wellman 1983; Wellman / Berkowitz 1988). In der ersten Auflage von Whites Identity and Control nimmt die Auseinandersetzung mit Rational Choice bereits größeren Raum ein (White 1992: 8f). In der Folge wird der Ton selbstbewusster: Mustafa Emirbayer formuliert 1997 den Anspruch auf ein eigenes >relationales< Paradigma. Auch bei Charles Tilly finden sich zahlreiche Passagen der Auseinandersetzung mit der erklärenden Handlungstheorie (Tilly 1998: 30ff; 2002: 33, 71f). Der Konflikt mit Rational Choice ist damit ein Teil der Erklärung für die starke Bedeutung, die die Phänomenologische Netzwerktheorie mittlerweile in den USA erlangt hat. ${ }^{3}$ Zudem ist er fester Bestandteil der >story< der PNT als der von ihren Autoren produzierten Selbststilisierung gegen die Rational Choice-Theorie.

\section{Netzwerk}

Nach der narrativen Betrachtung der Entstehung der Phänomenologischen Netzwerktheorie soll in diesem und im nächsten Abschnitt das Netzwerk der Autoren dieses Ansatzes genauer in den Blick genommen werden. Wie bereits aus dem Aufriss über die Entstehung deutlich wurde, bestehen enge persönliche Verbindungen zwischen den Autoren der PNT. Um die

3) In der deutschen Wissenschaftslandschaft deutet sich in den letzten Jahren eine Verbindung aus Systemtheorie und PNT an (Baecker 2005: 226ff; Holzer 2008). Die Attraktivität dieser Verbindung gründet sich wiederum in der gemeinsamen Frontstellung gegen die Rational Choice-Theorie. 
Struktur und das Ausmaß der internen Verflechtung der betrachteten Netzwerktheoretiker genauer zu untersuchen, soll im Folgenden eine formale Analyse des Netzwerks durchgeführt werden. Ziel soll es sein zu überprüfen, ob die Netzwerkstruktur die zwei eingangs formulierten Anforderungen an Invisible Colleges erfüllt. In diesem Abschnitt wird untersucht, ob die Autoren der PNT in einem dichten Netzwerk von persönlichen Austauschbeziehungen miteinander verknüpft sind (These 4). Die Frage der internen Differenzierung (These 5) wird im nächsten Abschnitt behandelt.

Netzwerke in der Wissenschaft wurden bisher vor allem mit Blick auf Zitationen oder auf Mitgliedschaften in Sektionen rekonstruiert (Small / Griffith 1974; Ennis 1992). Selten wurden eigene Befragungen durchgeführt (Mullins 1968; Breiger 1976; Mullins et al. 1977). Beide Methoden bringen gewisse Schwierigkeiten mit sich: Während Befragungen meist recht unvollständige Daten liefern, sind Zitationen oder gemeinsame Mitgliedschaften durchaus nicht immer Anzeichen für einen wissenschaftlichen Austausch. Zudem betont die PNT die Rolle von persönlichen Kontakten in Invisible Colleges (These 4), während Zitationen meist für eine eher unpersönliche Rezeption stehen. Wegen der Betonung von direkter Kommunikation wurden hier Ko-Autorschaften und Danksagungen in Büchern und Artikeln als Indikatoren für einen intellektuellen Austausch in einer persönlichen Beziehung zwischen zwei Autoren gewertet. Als Sample wurden mittels einer umfangreichen Literaturrecherche (unter Zuhilfenahme der Antworten aus der halbstandardisierten Befragung, die im Abschnitt 6 genauer vorgestellt wird) 18 Autoren identifiziert, die mit ihren Arbeiten als zur PNT zugehörig identifiziert wurden. ${ }^{4}$

Ko-Autorschaften als erster Indikator für einen direkten intellektuellen Austausch entstehen aus dem akademischen Austausch über theoretische Fragen, über Forschungsergebnisse oder auch bei deren gemeinsamer Erarbeitung. In der Soziologie sind Ko-Autorschaften allerdings sehr viel seltener als etwa in der Psychologie oder den Naturwissenschaften. So konnten zwischen den 18 zentralen Autoren der Phänomenologischen Netzwerktheorie lediglich sieben Ko-Autorschaften identifiziert werden. Dieser Befund deutet alleine nicht auf eine starke Vernetzung hin. Als zweiter Indikator für einen intellektuellen Austausch zwischen den Autoren wurden Danksagungen für Kritik oder wichtige Hinweise für eine Publikation heran gezogen. Während Zitationen eher einen distanzierten Einfluss anzeigen (und unter Umständen ja auch abgrenzend sein können), stehen Danksagungen und Ko-Autorschaften für einen direkten und kooperativen intellektuellen Austausch. Insofern weisen sie eher auf ein durch direkte Beziehungen geprägtes Invisible College hin als auf eine soziologische Schule im weiteren Sinne. ${ }^{5}$

Um auf der Basis dieser Danksagungen das Netzwerk der Netzwerktheorie zu rekonstruieren, habe ich Danksagungen von 48 Büchern und Artikeln der 18 zentralen Autoren der Phänomenologischen Netzwerktheorie aus den Jahren 1988 bis 2005 untersucht $^{6}$. Dabei fanden sich 149 Danksagungen an die jeweils übrigen 17 Autoren (von insgesamt 620). Damit fan-

4) Es liegt in der Natur von Netzwerken, dass mehr oder weniger willkürlich Analysegrenzen gezogen werden müssen. Natürlich muss dabei eine mögliche Verzerrung des Samples in Kauf genommen werden. Die Netzwerktheorie nimmt aber an, dass sich in der Analyse von notwendigerweise begrenzten Netzwerken bestimmte Strukturprinzipien aufspüren lassen (Martin 2003). Die Herein- oder Herausnahme von Netzwerkknoten dürfte an der Struktur des untersuchten Feldes wenig ändern.

5) Dabei werden Danksagungen oft auch strategisch eingesetzt, um im Diskurs von konkurrierenden Identitäten als Wissenschaftler einen höheren Status durch die eigene Platzierung neben den >GroBen< des Fachs oder durch den Nachweis von umfangreichem Sozialkapital zu symbolisieren (Hollstein / Schütze 2004). Im Sinne von Joel Podolny wirken die sozialen Beziehungen eines Akteurs hier als >Prisma<, durch das andere den eigenen Status einschätzen können (Podolny 2001).

6) Bei wenigen Artikeln mit zwei Autoren habe ich Danksagungen dem Erstautor zugerechnet, wenn der Zweitautor in der Auswahl der 18 wichtigsten Autoren der PNT nicht auftaucht. Die Daten können auf Anfrage zur Verfügung gestellt werden. 
den durchschnittlich 30,6 Prozent aller Danksagungen in den betrachteten Publikationen im Kreis der 18 Autoren statt. Im arithmetischen Mittel fanden sich in jedem der untersuchten Artikel oder Bücher 3,10 Danksagungen an die anderen wichtigsten Autoren der Netzwerktheorie. Diese Zahlen weisen auf einen regen intellektuellen Austausch zwischen den untersuchten 18 Autoren hin.

Als Minimal-Indikator für intellektuellen Austausch soll eine Ko-Autorschaft von zwei Autoren oder (mindestens) eine Danksagung eines Autors / einer Autorin an einen anderen Autor / eine andere Autorin gelten. Auf diese Weise ergeben sich 102 gerichtete Beziehungen zwischen den 18 Autoren (von 306 möglichen Ties). Im Folgenden sollen der Einfachheit halber die einfachen gerichteten Beziehungen als >Danksagungen< bezeichnet werden. Das Gesamtbild dieses Netzwerkes von intellektuellen Austauschbeziehungen zwischen den 18 zentralen Autoren der Phänomenologischen Netzwerktheorie ist in Abbildung 1 dargestellt.

Abbildung 1: Das Netzwerk von Danksagungen und Ko-Autorschaften zwischen den zentralen Autoren der Phänomenologischen Netzwerktheorie

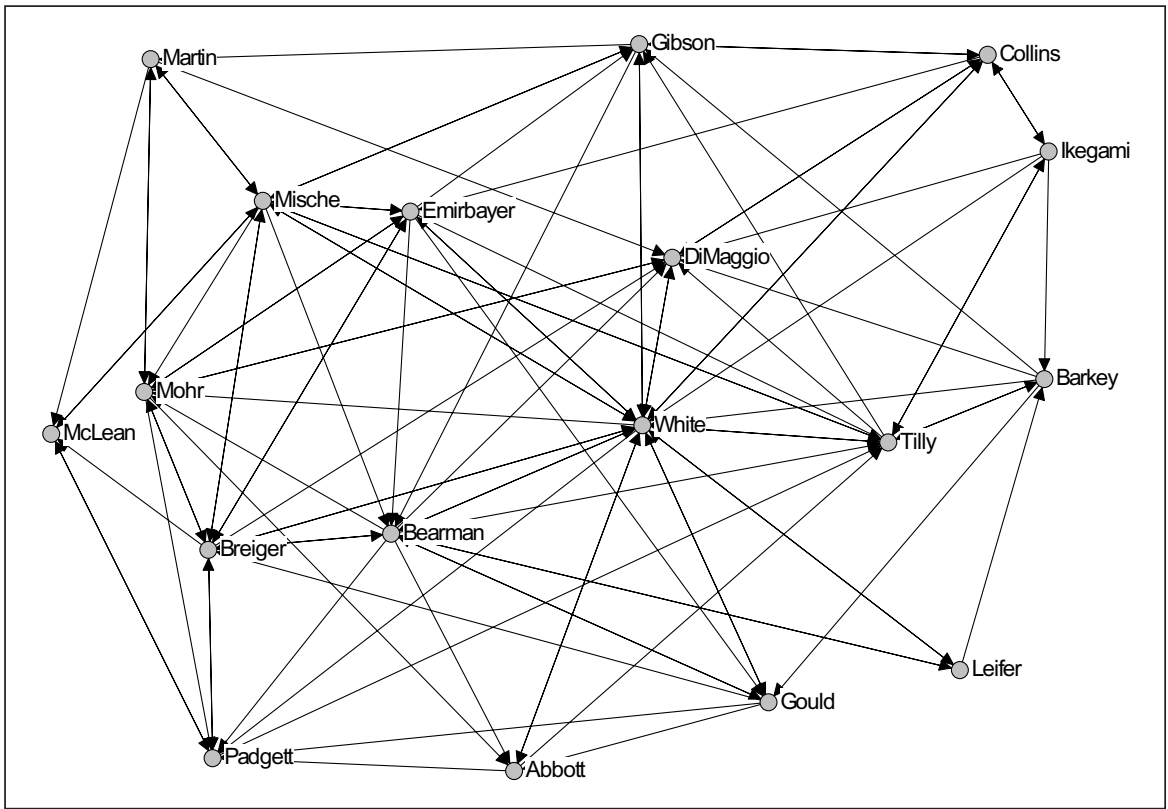

Ist dies nun ein Beleg für die hohe Dichte des Netzwerks der Netzwerktheoretiker im Sinne der These 4? Die Dichte des Netzwerks der gerichteten Danksagungen liegt für die betrachteten 18 Autoren bei 33,3 Prozent. Vergleichbare Zahlen fehlen leider, aber intuitiv erscheint dies eine relativ hohe Zahl. Dies gilt umso mehr, als 18 bereits eine recht große Zahl von Autoren ist, die zudem nicht in einen gemeinsamen institutionellen Rahmen eingebunden sind (auch wenn die Columbia University eine starke Rolle für das Netzwerk spielt). Eine genaue Analyse müsste zeigen, dass die betrachteten Danksagungen eine deutliche Verdichtung im Gesamtnetzwerk der Danksagungen in soziologischen Publikationen im betrachteten Zeitraum bilden - dies erscheint methodisch nur mit sehr hohem Aufwand machbar.

Nehmen wir als Nullhypothese an, dass die Danksagungen unter den amerikanischen Soziologen zufällig verteilt sind. Die American Sociological Association zählt 14.000 Mitglie- 
der, in den 44 Sektionen sogar 21.000. ${ }^{7}$ Bei 620 insgesamt ausgesprochenen Danksagungen wären unter 18 zufällig ausgewählten Mitgliedern der ASA und ihrer Sektionen 0,50 Danksagungen zu erwarten. Tatsächlich haben sich die betrachteten Autoren aber 149 Mal gedankt. Bei einer zufälligen Verteilung der Danksagungen unter amerikanischen Soziologen wäre eine solche Verdichtung deutlich unwahrscheinlicher als ein Lottogewinn. Die Nullhypothese einer völligen Zufälligkeit der Danksagungen kann damit verworfen werden. Die betrachteten Autoren bilden ein dichtes Netzwerk im akademischen Diskurs. Auch wenn der Vergleich mit anderen Netzwerkstrukturen hier nicht geleistet werden kann, lässt sich dies als Befund im Sinne der vierten These sehen.

\section{Zentrum / Peripherie oder Subgruppen?}

Auf der Ebene des Netzwerks muss noch die fünfte These betrachtet werden, die eine Differenzierung in Zentrum und Peripherie innerhalb des Netzwerkes vermutet. Dabei geht es um die Frage, inwiefern das Netzwerk kohäsive Subgruppen enthält oder durch ein Zentrum-Peripherie-Modell beschrieben werden kann. Wenn das Netzwerk in verschiedene Teilgruppen zerfiele, könnte man nicht sinnvoll von einem Invisible College sprechen. Es geht also letztlich um die Frage, ob das betrachtete Netzwerk der Struktur eines um einen personellen Kern kristallisierten sozialen Gebildes entspricht oder nicht.

Dafür wurde das Netzwerk der gerichteten Danksagungen mit UCINET 6.0 auf ZentrumPeripherie-Strukturen untersucht (Borgatti / Everett 1999). Dabei zeigte sich zwar keine perfekte, aber eine ausgeprägte Differenzierung in Kern und Peripherie, wie in Tabelle 1 dargestellt. In dieser Modellierung bilden Peter Bearman, Ronald Breiger, Paul DiMaggio, Mustafa Emirbayer, Ann Mische, John Mohr, Charles Tilly und Harrison White den personellen Kern mit einer internen Netzwerkdichte von 0,679. Andrew Abbott, Karen Barkey, Randall Collins, David Gibson, Roger Gould, Eiko Ikegami, Eric Leifer, John Levi Martin, Paul McLean und John Padgett sind dagegen in der Peripherie zu verorten. Sie sind untereinander nur mit einer Dichte von 0,167 verflochten. Wie zu erwarten ist diese Peripherie untereinander auch weniger dicht verbunden als mit dem Kern. ${ }^{8}$ Die Autoren in der Peripherie gruppieren sich um den Netzwerkkern und nicht als separater Netzwerkcluster. Die Güte dieser Modellierung der Netzwerkstruktur lässt sich als Korrelation der beobachteten Struktur mit einer perfekten Kern-Peripherie-Differenzierung angeben. Diese beträgt 0,518 (Pearsons r). ${ }^{9}$ Es zeigt sich, dass das Netzwerk der PNT recht deutlich einem Zentrum-Peripherie-Modell entspricht.

7) Quelle: Homepage der ASA: http://www.asanet.org/page.ww?section=About+ASA\&name=About+ ASA, abgerufen am 19.11.2007.

8) Das Netzwerk der Danksagungen aus der Peripherie in den Kern weist eine Dichte von 0,313 auf. Danksagungen aus dem Kern in die Peripherie finden sich mit einer Dichte von 0,300 in einer ähnlichen Größenordnung.

9) Für die Korrelation werden nur die Beziehungen innerhalb des Kerns und die in der Peripherie mit dem Ideal eines vollkommen verdichteten Kerns und einer Peripherie ohne jede interne Verflechtung verglichen. Die Beziehungen zwischen Kern und Peripherie werden nicht betrachtet, weil sich dafür keine Vorhersagen treffen lassen (Borgatti / Everett 1999: 381, 383). 
Tabelle 1: Kern-Peripherie-Differenzierung (kategorial)

\begin{tabular}{|c|c|c|c|c|c|c|c|c|c|c|c|c|c|c|c|c|c|c|}
\hline & 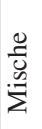 & 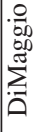 & 苞 & 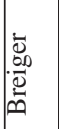 & $\frac{\stackrel{9}{3}}{3}$ & $\gtrsim$ & 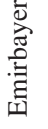 & $\frac{z}{\sum^{\circ}}$ & $\begin{array}{l}\text { dे } \\
\text { 产 } \\
\ddot{\oplus}\end{array}$ & 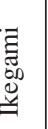 & 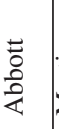 & 䏤 & 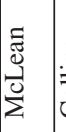 & $\stackrel{\tilde{\Xi}}{\overline{0}}$ & 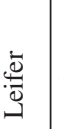 & 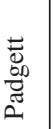 & 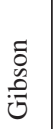 & $\begin{array}{l}\frac{}{\vec{z}} \\
\stackrel{0}{0}\end{array}$ \\
\hline Mische & $\mathrm{X}$ & & & 1 & 1 & 1 & 1 & 1 & & & & 1 & 1 & & & & 1 & \\
\hline DiMaggio & & $\mathrm{X}$ & & & 1 & & & 1 & & & & & & & & & & \\
\hline Bearman & & 1 & $\mathrm{X}$ & 1 & 1 & 1 & & 1 & & & 1 & & & & 1 & 1 & & 1 \\
\hline Breiger & 1 & 1 & 1 & $\mathrm{X}$ & 1 & & 1 & 1 & & & & & 1 & & & 1 & & \\
\hline White & 1 & 1 & 1 & 1 & $\mathrm{X}$ & 1 & 1 & 1 & & & 1 & & & 1 & 1 & 1 & 1 & 1 \\
\hline Tilly & 1 & 1 & & & 1 & $\mathrm{X}$ & & & 1 & 1 & & & & & & & 1 & \\
\hline Emirbayer & 1 & & 1 & 1 & 1 & 1 & $\mathrm{X}$ & 1 & & & & & & & & & 1 & 1 \\
\hline Mohr & & 1 & & 1 & . & & 1 & $\mathrm{X}$ & & & 1 & 1 & & & & 1 & & \\
\hline Barkey & & 1 & & & 1 & 1 & & & $\mathrm{X}$ & & & & & & & & 1 & 1 \\
\hline Ikegami & & 1 & & & 1 & 1 & & & 1 & $\mathrm{X}$ & & & & 1 & & & & \\
\hline Abbott & & & & & 1 & 1 & & & & & $\mathrm{X}$ & & & & & 1 & & \\
\hline Martin & 1 & 1 & & & & & & 1 & & & & $\mathrm{X}$ & 1 & & & & & \\
\hline McLean & 1 & & & & & & & & & & & & $\mathrm{X}$ & & & 1 & & \\
\hline Collins & & 1 & & & 1 & & 1 & & & 1 & & & & $\mathrm{X}$ & & & 1 & \\
\hline Leifer & & & 1 & & 1 & & & & 1 & & & & & & $\mathrm{X}$ & & & \\
\hline Padgett & & & & 1 & & 1 & & & & & & & 1 & & & $\mathrm{X}$ & & \\
\hline Gibson & 1 & & 1 & & 1 & & & & & & & 1 & & 1 & & & $\mathrm{X}$ & \\
\hline Gould & & & 1 & 1 & 1 & & & & & & 1 & & & & & 1 & & $\mathrm{X}$ \\
\hline
\end{tabular}

Dabei sollte man nicht den Fehler machen, die Autoren in der Peripherie generell für weniger wichtig als die im Zentrum zu halten. Zentrale Autoren haben zwar einen größeren Einfluss innerhalb des Netzwerkes. Stephan Fuchs zufolge wird im Kern eines Netzwerks dessen Identität definiert (Fuchs 2001: 281ff). Bei soziologischen Schulen werden hier Theorie und Methoden festgelegt, an denen sich das gesamte Netzwerk orientiert. Dagegen überschneiden sich in der Peripherie Einflüsse aus verschiedenen Netzwerkkontexten. Die Peripherie ist damit für den Umweltkontakt zuständig. Hier werden Irritationen aufgenommen, die schließlich in das Netzwerk inkorporiert oder auch als irrelevant verworfen werden. Insofern leistet die Peripherie wichtige und unerlässliche Aufgaben für ein Netzwerk, auch wenn das Zentrum stärker im Mittelpunkt der Aufmerksamkeit steht. Die Aufnahme von Umwelteinflüssen in der Peripherie bewahrt die Theorie vor zu viel Isolation und Redundanz. Forscher wie Andrew Abbott oder Randall Collins sitzen an den Schnittstellen zwischen der PNT und anderen Theoriekontexten. Wie Ronald Burt schreibt: 
»people who stand near the holes in a social structure are at higher risk of having good ideas.« (Burt 2004: 349)

Im Zentrum des betrachteten Netzwerks steht vor allem Harrison White. White fungiert als der >intellectual leader< der Phänomenologischen Netzwerktheorie, an dem sich das Netzwerk orientiert (Griffith / Mullins 1972: 961). Im Sinne von Randall Collins wirkt White für die Gruppe wie ein >Durkheimian sacred object< (Collins 2004: 36ff). Ein Autor wird zum zentralen Referenzpunkt, obwohl seine Arbeit sowohl in ihrer Entstehung als auch in ihrer Wirkung im Netzwerkkontext gründet. Um ihn und seine Arbeiten gruppiert sich das Netzwerk - er wird zum Bezugspunkt für die >Involution< des Netzwerks.

Eine alternative Interpretation wäre, dass die Differenzierung in Zentrum und Peripherie im Wesentlichen Statusunterschiede widerspiegelt. Diese Interpretation liegt nahe, da mit Peter Bearman, Ronald Breiger, Paul DiMaggio, Charles Tilly und Harrison White sehr renommierte Soziologen im Zentrum des Netzwerks zu finden sind. Andererseits tauchen dort auch Ann Mische und Mustafa Emirbayer auf, die während der fraglichen Zeit vor allem als Assistant Professors tätig waren. Zugleich befinden sich mit Andrew Abbott, Randall Collins und John Padgett drei schon damals sehr wichtige Professoren in der amerikanischen Soziologie in der Peripherie. Dies weist darauf hin, dass eher inhaltliche Gesichtspunkte als Statusunterschiede über die Einordnung in Zentrum und Peripherie entscheiden - dass also die Autoren im Zentrum sich stärker an der Domäne von theoretischen Annahmen und Konzepten der PNT orientieren. Wie im nächsten Abschnitt zu sehen sein wird, identifizieren sich die Autoren im Zentrum auch stärker mit der PNT als jene in der Peripherie.

Doch die fünfte These vermutet nicht nur, dass das Netzwerk in Kern und Peripherie differenziert ist, sondern auch, dass es nicht in Subgruppen aufzuteilen ist. Um dies zu untersuchen, wurde mit UCINET 6.0 eine Analyse von Fraktionen im Netzwerk der gerichteten Danksagungen zwischen den 18 zentralen Autoren der Phänomenologischen Netzwerktheorie durchgeführt. Der Factions-Algorithmus teilt das Netzwerk in Subgruppen auf, die intern stark verdichtet sind und nach außen gering (Hanneman / Riddle 2005, Kapitel 11). Aus mehreren möglichen Modellierungen wurde schließlich ein Modell mit vier Subgruppen ausgewählt, das relativ gute Erklärungskraft beweist und sich gut interpretieren lässt (Tabelle 2). Dieses Modell soll hier kurz vorgestellt werden. In diesem Modell zeigen die vier Subgruppen jeweils interne Dichten zwischen 0,60 und 1,00. Also finden sich innerhalb der Subgruppen mindestens 60 Prozent der möglichen Ties. Dagegen finden sich nur 23,1 Prozent der möglichen Verbindungen zwischen den Subgruppen. Insgesamt werden durch diese Einteilung in vier Fraktionen 226 von 306 Einzelbeziehungen (also 73,9\%) richtig vorhergesagt. 
Tabelle 2: Modell des Netzwerks mit vier Fraktionen

\begin{tabular}{|c|c|c|c|c|c|c|c|c|c|c|c|c|c|c|c|c|c|c|}
\hline & 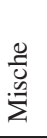 & $\sum^{ \pm}$ & 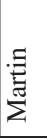 & 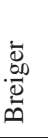 & 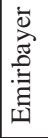 & 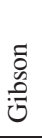 & $\begin{array}{l} \pm \\
0 \\
0 \\
0 \\
\end{array}$ & 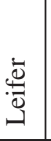 & $\begin{array}{l}\text { 믐 } \\
\text { ن }\end{array}$ & $\stackrel{\mathscr{B}}{3}$ & 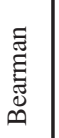 & 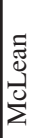 & 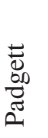 & 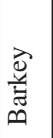 & 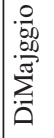 & 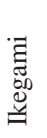 & $\vec{\Xi}$ & $\frac{\mathscr{g}}{\bar{\theta}}$ \\
\hline Mische & $\mathrm{X}$ & 1 & 1 & 1 & 1 & 1 & & & & 1 & 1 & 1 & & & & & 1 & \\
\hline Mohr & & $\mathrm{X}$ & 1 & 1 & 1 & & 1 & & & & & & 1 & & 1 & & & \\
\hline Martin & 1 & 1 & $\mathrm{X}$ & & & & & & & & & 1 & & & 1 & & & \\
\hline Breiger & 1 & 1 & & $\mathrm{X}$ & 1 & & & & & 1 & 1 & 1 & 1 & & 1 & & & \\
\hline Emirbayer & 1 & 1 & & 1 & $\mathrm{X}$ & 1 & & & 1 & 1 & 1 & & & & & & 1 & \\
\hline Gibson & 1 & & 1 & & & $\mathrm{X}$ & & & & 1 & 1 & & & & & & & 1 \\
\hline Abbott & & & & & & & $\mathrm{X}$ & & & 1 & & & 1 & & & & 1 & \\
\hline Leifer & & & & & & & & $\mathrm{x}$ & & 1 & 1 & & & 1 & & & & \\
\hline Gould & & & & 1 & & & 1 & & $\mathrm{X}$ & 1 & 1 & & 1 & & & & & \\
\hline White & 1 & 1 & & 1 & 1 & 1 & 1 & 1 & 1 & $X$ & 1 & & 1 & & 1 & & 1 & 1 \\
\hline Bearman & & 1 & & 1 & & & 1 & 1 & 1 & 1 & $\mathrm{Y}$ & & 1 & & 1 & & 1 & \\
\hline McLean & 1 & & & & & & & & & & & $\mathrm{X}$ & 1 & & & & & \\
\hline Padgett & & & & 1 & & & & & & & & 1 & $\mathrm{X}$ & & & & 1 & \\
\hline Barkey & & & & & & 1 & & & 1 & 1 & & & & $\mathrm{X}$ & 1 & & 1 & \\
\hline DiMaggio & & 1 & & & & & & & & 1 & & & & & $\mathrm{X}$ & & & 1 \\
\hline Ikegami & & & & & & & & & & 1 & & & & 1 & 1 & $\mathrm{X}$ & 1 & 1 \\
\hline Tilly & 1 & & & & & 1 & & & & 1 & & & & 1 & 1 & 1 & $\mathrm{X}$ & \\
\hline Collins & & & & & 1 & 1 & & & & 1 & & & & & 1 & 1 & & $\mathrm{X}$ \\
\hline
\end{tabular}

Die erste Subgruppe (mit einer Dichte von 63,3\%) besteht aus den zumeist noch relativ jungen Netzwerkforschern Ann Mische, ihrem Lebenspartner David Gibson, Mustafa Emirbayer sowie John Mohr und John Levi Martin. Diese Forscher haben ihre PhDs in den Jahren 1989 bis 1999 abgeschlossen und waren gegen Ende des betrachteten Zeitraums als Associate oder Assistant Professors an renommierten Universitäten in den USA tätig (Mustafa Emirbayer und John Levi Martin sind inzwischen volle Professoren in Wisconsin-Madison bzw. Berkeley). $\mathrm{Zu}$ dieser jungen Generation gruppiert sich noch Ronald Breiger, der bereits in den 70ern mit Harrison White die Blockmodell-Analyse entwickelte. Ronald Breiger hat mit seiner Theorie der Dualität von Akteuren und Netzwerkstrukturen wichtige Anstöße für die Arbeiten dieser Subgruppe geliefert (Breiger 1974; 2000). Allgemein zeichnen sich diese Autoren durch ihr Interesse an der kreativen Weiterentwicklung der Theorie aus - vor allem in Richtung der Anwendung auf die phänomenologische Ebene. 
Harrison White selbst ist mit seinem Columbia-Kollegen Peter Bearman und ihren beiden am engsten verbundenen Studenten, dem 2002 verstorbenen Roger Gould und dem in den 90ern aus der akademischen Laufbahn ausgeschiedenen Eric Leifer in der zweiten Subgruppe zu finden. In dieser Gruppe taucht auch Andrew Abbott - Professor in Chicago und seit 2000 Herausgeber des American Journal of Sociology - als relativer Außenseiter im Netzwerk der Netzwerktheoretiker auf. Diese Gruppe wirkt in der theoretischen Ausrichtung eher heterogen und ist vor allem durch Professor-Studenten-Beziehungen geprägt. Sie hat eine interne Dichte von 70,0 Prozent.

Die dritte Gruppe besteht nur aus John Padgett und Paul McLean. Dieser Gruppe wäre auch Christopher Ansell zuzuordnen, der aus methodischen Gründen nicht im Sample auftaucht. McLean und Ansell haben bei John Padgett, einem Politikwissenschaftler an der University of Chicago, promoviert. Padgett, Ansell und McLean erarbeiten seit langem gemeinsam Studien über Verbindungen zwischen Patrizierfamilien im Florenz der Renaissance. Die bekannteste Arbeit aus dieser Subgruppe ist die berühmte Netzwerkstudie zur Machtergreifung der Medici von Padgett und Ansell (Padgett / Ansell 1993). Padgett und McLean treten nicht nur als Ko-Autoren auf, sondern danken sich auch gegenseitig in anderen Artikeln. Diese Netzwerkfraktion weist damit eine Dichte von 1 auf.

Viertens identifiziert das Modell die Gruppe von Karen Barkey, Paul DiMaggio, Eiko Ikegami, Charles Tilly und Randall Collins (interne Dichte: 60,0\%). Barkey, Ikegami und Tilly sind in New York angesiedelte Historische Soziologen. Der Neo-Institutionalist und Kultursoziologe DiMaggio von der Princeton University ist ein Schüler von White. Der Theoretiker Collins (University of Pennsylvania) kam dagegen eher von außen zur PNT. Beide haben starke Verbindungen zu der Gruppe um Tilly. Auch diese Gruppe hat damit einen gemeinsamen thematischen Fokus in der Historischen Soziologie, ist aber zugleich so heterogen, dass etwa die Zugehörigkeit von Randall Collins und Paul DiMaggio nicht ohne weiteres interpretiert werden kann. Hier wie an anderen Stelle erscheint die Zuordnung von einzelnen Autoren zu Subgruppen nicht unbedingt zwingend - das Modell ordnet aber alle Autoren einer Subgruppe zu.

Das Modell identifiziert also vier Fraktionen mit erhöhter interner Dichte, die sich zudem durch Gemeinsamkeiten wie Arbeitsschwerpunkte oder universitäre Affiliation auszeichnen. Wie ist dieses Modell - im Gegensatz zum zuvor präsentierten Zentrum-Peripherie-Modell - insgesamt zu bewerten? Das Modell bildet vier reale Verdichtungen innerhalb des Netzwerks ab, die auf eine Tendenz zur Differenzierung in Einzeldiskurse hinweisen. Allerdings bildet auch dieses Modell das Netzwerk nicht perfekt ab. Die Güte der beiden Modellierungen lässt sich an dem Anteil der Varianz bemessen, der mit ihnen jeweils erklärt wird. Das Zentrum-Peripherie-Modell liefert für den Bereich, für den es Vorhersagen macht (die Beziehungen innerhalb des Kerns und innerhalb der Peripherie) eine Erklärungskraft von 26,8 Prozent $\left(\mathrm{R}^{2}\right)$ der Varianz der tatsächlichen Ties. Dies ist aber nur ein Teil des gesamten Netzwerkes. Nimmt man die Beziehungen zwischen Zentrum und Peripherie hinzu (über die das Modell keine Aussagen macht), reduziert sich die ausgeschöpfte Varianz auf 13,3 Prozent (bei einem Pearsons $r$ von 0,365). Das Modell der vier Subgruppen macht dagegen von vornherein Vorhersagen für alle Beziehungen und liefert dabei eine Erklärungskraft von 14,1 Prozent $\left(\mathrm{R}^{2}\right.$; Pearsons $\left.\mathrm{r}=0,376\right)$.

Beide Modelle erklären also - nahezu unabhängig voneinander - einen etwa gleich großen Anteil an der gesamten Varianz von Danksagungen und Ko-Autorschaften (von jeweils einem Siebtel). Allerdings werden dabei gewissermaßen Äpfel mit Birnen verglichen. So ist die Einteilung in vier Subgruppen sehr viel feiner als eine kategoriale Unterscheidung zwischen Zentrum und Peripherie. In einem Fall liefert eine einzige dichotome Variable eine erklärte Varianz von 13,3 Prozent - und dass, obwohl das Modell nur für 160 der 306 mögli- 
chen Ties Vorhersagen macht. Im anderen Fall erklärt eine Aufteilung in vier Gruppen 14,1 Prozent der Varianz. Insofern wäre die Zentrum-Peripherie-Differenzierung das bessere weil einfachere - Modell mit einer nahezu gleich großen Erklärungskraft. Sinnvoll vergleichen ließe sich das Zentrum-Peripherie-Modell am ehesten mit einem Modell mit zwei Subgruppen. Ein solches liefert für das betrachtete Netzwerk aber nur eine ausgeschöpfte Varianz von 7,2 Prozent (und wurde wegen dieser geringen Aussagekraft hier nicht eingehender vorgestellt). ${ }^{10}$

Vor diesem Hintergrund der Maßzahlen für die Güte der beiden Modelle lässt sich die fünfte These (bei aller methodisch gebotenen Vorsicht) tendenziell be-stätigen. Wir finden im Netzwerk der betrachteten Autoren eine deutliche Differenzierung in Zentrum und Peripherie. Mit dieser Differenzierung in Kern und Peripherie zeigen sich Anzeichen einer Involution auf der Netzwerkebene - ein Hinweis darauf, dass die Phänomenologische Netzwerktheorie tatsächlich ein Invisible College bildet. Daneben lassen sich auch verdichtete Subgruppen identifizieren. Zentrum-Peripherie-Differenzierung und Subgruppenbildung prägen gemeinsam als Strukturprinzipien das betrachtete Netzwerk. Wie Harrison White betont: Die soziale Wirklichkeit ist >messy< und ähnelt eher den polymeren Molekülen von Gelen als perfekt geordneten Kristallen (White 1992: 70, 342). Die Aufteilung in Subgruppen ist eher geringer ausgeprägt als die Differenzierung in Zentrum und Peripherie. Insgesamt lässt sich deswegen die Ausgangsfrage, ob die PNT ein Invisible College bildet, auf der Ebene der Netzwerkstrukturen mit einem vorsichtigen $>\mathrm{Ja}<$ beantworten.

Natürlich darf man die Netzwerkdaten nicht überbewerten. Viele von ihnen entstehen aus gemeinsamer Affiliation an den großen amerikanischen Departments heraus (vor allem an der Columbia University, aber auch in Chicago) oder sind sogar Professor-Studenten-Beziehungen. Andererseits lässt sich im Sinne der PNT argumentieren, dass aus solchen engen Beziehungen heraus gemeinsame Deutungsmuster entstehen - und dass die Frage der gemeinsamen inhaltlichen Orientierung auch entscheidend dafür ist, welche Beziehungen sich stabilisieren und welche nicht. So kamen etwa Mustafa Emirbayer, Randall Collins und John Levi Martin von außen in das Netzwerk. Auf der Ebene der Netzwerkstruktur zeigen sich Anzeichen für ein Invisible College der betrachteten Autoren. Entscheidend für die Frage, ob sich dahinter ein gemeinsamer theoretischer Ansatz verbirgt, ist aber die inhaltliche Ebene der theoretischen Orientierung der Autoren (These 1) und deren gemeinsamer Identifikation (These 6).

\section{Identität}

Nach der Struktur des Netzwerks soll nun die Ebene der Sinnformen in den Blick genommen werden. Dabei geht es um die Frage, inwiefern die betrachteten Forscher tatsächlich der $>$ Phänomenologischen Netzwerktheorie`zuzurechnen sind, also: inwiefern sie Anhänger eines gemeinsamen Forschungsprogramms sind und sich auch selbst als solche begreifen. Dies betrifft die sechste These: Gibt es eine >kollektive Identität< der PNT? Und taucht sie als Label in den intellektuellen Auseinandersetzungen in der Wissenschaftslandschaft auf? Um diese Frage zu beleuchten, greife ich erstens auf neuere Publikationen zurück, die sich mit der Netzwerkforschung befassen. Zweitens habe ich die zentralen Autoren des Ansatzes zwischen September 2005 und April 2006 direkt oder per e-mail mittels eines kurzen halbstandardisierten Fragebogens befragt. Den folgenden Betrachtungen liegen Antworten von 14 der oben aufgeführten 18 zentralen Autoren zugrunde.

10) Die maximale Erklärungskraft findet sich bei einer Aufteilung in sieben Subgruppen $\left(R^{2}=19,3 \%\right)$. Dieses Modell wurde hier nicht vorgestellt, weil es komplexer, aber nicht wesentlich besser ist als das Modell mit vier Gruppen. 
Als Erstes fragte ich nach der Meinung der Befragten, ob eine Phänomenologische Netzwerktheorie in den USA als soziologisch-theoretische Schule existiert oder nicht. Dabei ist das Label aber selbst nicht weit verbreitet und deswegen erklärungsbedürftig. ${ }^{11}$ Deswegen verwendete ich zwei Stimuli: Im Anschreiben zog ich die Verbindung zu Harrison White. Und in der Frage definierte ich, dass die PNT von der Annahme ausgeht, dass symbolische Formen (Kategorien, Identitäten, Frames, Schemata) und die Struktur sozialer Netzwerke sich wechselseitig beeinflussen. Die zweite Frage ging danach, ob die Autoren ihre Arbeit als Teil der so definierten PNT sehen. Nur diese ersten beiden Fragen sollen hier betrachtet werden, weil diese relevant für die Diskussion der sechsten These einer >Identität< der PNT sind.

Die erste Frage nach der Existenz der Phänomenologischen Netzwerktheorie als eigenem Ansatz wurde von zehn der vierzehn Befragten eindeutig bejaht. Allerdings antworteten auch zwei der Autoren skeptisch bis verneinend: John Padgett zeigte erst seine Unkenntnis des Labels und grenzte sich später deutlich von einer zu starken Betonung des Kulturellen (wie bei John Mohr oder Ronald Breiger) ab. Er sah sich eher als Anhänger des klassischen Strukturalismus (e-mails vom 25.4.06). Andrew Abbott dagegen antwortete:

$» I$ don't know whether PNT exists as a social entity [...] since I don't know whether there is such a tradition I can't say whether I'm in it. If your question is whether my work has been influenced by Harrison White, then the answer is yes, certainly.« (e-mail vom 7.4.2006)

Nicht zufällig sind diese beiden Autoren eher in der Peripherie des Netzwerkes angesiedelt (und weisen auch geographisch an der University of Chicago eine gewisse Distanz zum Kern im Gebiet um New York auf). Diese beiden Antworten verweisen auf zwei Probleme, die auch in den anderen Interviews auftauchten. Erstens fehlt es an einem eindeutigen und gängigen Label für den Theorieansatz. Zweitens finden sich - wie bereits angedeutet - in dem Netzwerk unterschiedliche Richtungen, bei denen nicht ganz klar sind, ob sie eigentlich einem Ansatz zuzurechnen sind. John Padgett etwa verfolgt eher ein klassisch strukturalistisches Programm, in dem soziale Vorgänge (und kulturelle Formen) auf Strukturen in Netzwerken zurückgeführt werden. Andere Autoren wie Harrison White, Paul McLean, Randall Collins oder Ann Mische fokussieren stärker auf das Zusammenspiel von Sinnformen und Netzwerkstrukturen.

So wichtig diese Unterschiede teilweise sein mögen, so deutlich sind aber auch die Gemeinsamkeiten des Ansatzes. Die meisten Autoren ließen keinen Zweifel daran, dass sie sich als Mitglieder einer Theorierichtung begreifen. Stellvertretend für viele steht die Antwort von John Levi Martin:

$»$ Yes I think there is increasing congealing in this direction. It's still unsystematized, and is half between an insight and a longing. People are putting these things together in different, often incompatible, ways. But there is excitement that something can happen here." (e-mail vom 30.3.2006)

In diesem Sinne gaben fast alle Befragten auf die zweite Frage hin an, ihre Arbeiten zumindest teilweise als Teil der PNT zu sehen. Peter Bearman, Mustafa Emirbayer, Eiko Ikegami, John Mohr, Charles Tilly und Harrison White sahen die meisten oder alle ihrer Arbeiten als der PNT zugehörig. Ronald Breiger, Randall Collins, David Gibson und John Levi Martin antworteten mit >partly<, während Paul DiMaggio angab, seine Arbeit sei von der

11) Zum Hintergrund der Bezeichnung: Ronald Breiger (Breiger 1976: 118), Harrison White (White 1992: 65), und Eiko Ikegami (Ikegami 2000: 995ff), aber auch Gary Alan Fine und Sherryl Kleinman (Fine / Kleinman 1983: 102) schreiben, dass Netzwerke >phänomenologisch< konstituiert sind - und dass es in ihren Arbeiten eben um die Erforschung des Wechselspiels von phänomenologischem Sinn und der Struktur von Netzwerken geht. Auf die Frage nach dem Ursprung des Labels antwortete Harrison White: »I don't know who made it up, but >phenomenological network theory< captures what has always been my own interest and approach.« (e-mail vom 19.3.2005) 
PNT >beeinflusst $<$. Nur John Padgett und Andrew Abbott verneinten sie eher - in Folge ihrer Verneinung der ersten Frage (s.o.). ${ }^{12}$ Diese Selbstzuordnung zur PNT lässt sich als Anzeichen für eine gemeinsame Identität der PNT sehen, aber auch als Hinweis auf eine gemeinsame theoretische Ausrichtung im Sinne der ersten These. Dieser Sinn für Gemeinsamkeit lässt sich sicher nicht nur auf ähnliche theoretische Annahmen zurückführen, sondern gerade auch auf die starke Vernetzung zwischen den Autoren. Wiederkehrende (kooperative) Interaktion führt zu einer gemeinsamen Weltsicht und zu einer gemeinsamen Identität (Erickson 1988; Gould 1995; Ansell 1997).

Allerdings fällt es schwer, eine eindeutige Identität des Theorieansatzes festzumachen. Wie bereits angedeutet, ist >Phänomenologische Netzwerktheorie< kein gängiges Label (wie sich auch aus fast allen Antworten lesen lässt). Ronald Breiger fasst die Arbeiten des Ansatzes als »network thinking about culture and cognition« zusammen (Breiger 2004: 519ff). John Mohr bezeichnet ihn als »new structuralist project in cultural analysis« (Mohr 2000). Aber Mohr geht es eher um die netzwerkanalytische Aufdeckung von Tiefenstrukturen in der Kultur (Mohr 1998) als um das Wechselspiel zwischen sozialen Netzwerken und kulturellen Deutungsmustern. Mustafa Emirbayer zufolge wurde das Theorieprojekt eine Zeit lang als »New York School of Network (or Relational) Sociology « bezeichnet (e-mail vom 23.2.2006). Dieser Name bezog sich auf die regionale Konzentration vieler Autoren des Ansatzes in der New York Metropolitan Area. Gerade in der zweiten Hälfte der 90er Jahre leiteten Harrison White an der Columbia University und Mustafa Emirbayer an der New School for Social Research einige Theorie-Workshops, die als Treffpunkte für viele Autoren aus dem Bereich und von vielen Universitäten aus der Gegend in und um New York dienten (email von Ann Mische vom 15.4.2006). ${ }^{13}$ Da das von Emirbayer angeführte Label aber in keiner anderen Antwort auftauchte, ist es wohl heute kaum noch gebräuchlich. Charles Tilly schließlich äußerte, dass bisher eher der Begriff >Relational Realism< gebräuchlich wäre, wie es etwa bei Margaret Somers auftaucht (Persönliches Gespräch am 15.9.2005; Somers 1998).

In der Fremdbeschreibung tauchen wiederum neue Labels auf: So fassen die britischen Anthropologen Hannah Knox, Mike Savage und Penny Harvey die Arbeiten von White und Mische als >cultural approach to social networks< oder als >new cultural sociology of networks< (Knox / Savage / Harvey 2006: 121, 129). Boris Holzer dagegen sieht die Theorie Harrison White als >relationalen Konstruktivismus< (Holzer 2006: 79ff). Dieses Label steht in scheinbarem Widerspruch zu dem von Tilly angeführten >relationalen Realismus': Soziale Strukturen sind symbolisch konstruiert, aber dadurch auch real.

Diese Labelling-Probleme verweisen darauf, dass eine eindeutige Identität für das hier betrachtete Theorie-Netzwerk bisher nicht etabliert ist. Stattdessen sind heterogene Versuche zu beobachten, den Ansatz auf die eine oder andere Weise begrifflich zu fassen und als Identität in der Wissenschaftslandschaft zu etablieren. Darin erinnert der Ansatz an den Symbolischen Interaktionismus, der erst sehr spät von Herbert Blumer als Theorieansatz kodifiziert und mit seinem Label versehen wurde (Fine 1995). Möglicherweise wird es im Falle der

12) Zwischen dieser Selbstzuordnung von >no< (0) bis >yes< (4) und der dichotomen Einteilung in Zentrum und Peripherie ergibt sich eine bivariate Korrelation von 0,479 (Pearsons r) bei einer Signifikanz von 0,098 (trotz der sehr geringen Fallzahl von 13 betrachteten Autoren). Autoren im Zentrum der PNT identifizieren sich also tendenziell auch eher mit dieser. Dies lässt sich als vorsichtiger Hinweis darauf verstehen, dass im Zentrum dieses sozialen Gebildes tatsächlich deren Identität definiert und reproduziert wird (Fuchs 2001: 281ff).

13) Wie bei anderen Labels für Theorieschulen (Frankfurter Schule, Bristol School of Social Psychology, Chicago School) unterstreicht hier der regionale Bezug des Labels die Wichtigkeit von direkter Interaktion für die Theorieentwicklung (Mullins 1973: 29). 
PNT nie eine solche Stabilisierung von Erwartungen um ein etabliertes Label geben, weil die Arbeiten der Autoren trotz der gemeinsamen Annahmen und Begriffe doch recht heterogen sind. Das Netzwerk der PNT wird eher durch den engen akademischen Austausch und die daraus resultierende Ähnlichkeit der Perspektive zusammengehalten als durch ein abstraktes symbolisches Label. Die Identität liegt damit mehr auf der >praktischen< als auf der theoretischen Ebene. In einem ähnlichen Sinne hat Christopher Ansell argumentiert, dass die französische Arbeiterbewegung eher durch die kooperative Praxis der Streikbörsen geeint wurde als durch ein gemeinsames ideologisches Programm (Ansell 1997). Im Sinne der Bourdieuschen Habitustheorie werden Identität und Differenz in erster Linie >praktisch< konstruiert, nicht auf abstrakter symbolischer Ebene.

\section{Schluss}

Insgesamt lässt sich damit die Frage nach der Existenz einer >Phänomenologischen Netzwerktheorie< als Invisible College nicht eindeutig mit >ja< oder >nein< beantworten. Stattdessen muss die Diagnose stärker differenziert werden: Auf der Ebene der Netzwerkstruktur finden sich deutliche Anzeichen eines Invisible College. Die Danksagungen und KoAutorschaften der 18 zentralen Autoren der PNT bilden ein dichtes Netzwerk im akademischen Diskurs (Abschnitt 4). Dieses Netzwerk zeigt zwar eine gewisse Tendenz zur Ausbildung von Subgruppen, ist aber insgesamt eher in Zentrum und Peripherie differenziert (Abschnitt 5). Autoren wie Peter Bearman, Ronald Breiger, Paul DiMaggio, Ann Mische, Charles Tilly und vor allem Harrison White bilden den Kern eines Netzwerks, um den herum sich eine Peripherie von Nachwuchsforschern und von renommierten Professoren (wie Randall Collins, John Padgett und Andrew Abbott) als Bindegliedern zu anderen Netzwerkkontexten gruppiert. Dies entspricht dem Bild eines involuierten Netzwerkes, das um einen zentralen Fokus herum kristallisiert.

Auf der Sinnebene fällt das Urteil weniger eindeutig aus. Zwar zeichnet sich das Netzwerk durch eine gemeinsame Forschungsperspektive (mit theoretischen Annahmen und Konzepten) und auch durch einen durch die verdichtete Interaktion entstandenen Sinn für Gemeinsamkeit aus (Abschnitte 1 und 6). Der Ansatz kombiniert die strukturalistische Perspektive der Netzwerkanalyse mit dem Interesse an der Phänomenologie von Sinnformen aus der Verstehenden Soziologie (Abschnitt 2). Der Sinn für Gemeinsamkeit wird insbesondere durch die Auseinandersetzung mit der Rational Choice-Theorie gestärkt (Abschnitt 3). Allerdings fehlt es an einem eindeutigen Label für den Ansatz, an dem sich Erwartungen auch im übergeordneten Netzwerk der soziologischen Wissenschaftslandschaft festmachen könnten (Abschnitt 6). Deswegen taucht die PNT als Identität im Wissenschaftsdiskurs bisher wenig auf.

Diese Befunde erscheinen nur auf den ersten Blick widersprüchlich. Im Sinne der Theorie kann man nie von einer perfekten Ordnung der sozialen Realität auf der Ebene der symbolischen Beschreibungen oder der Netzwerkstrukturen ausgehen. Die soziale Wirklichkeit besteht nicht aus eindeutig abgrenzbaren Entitäten, deren Einheit unproblematisch und gegeben wäre. Stattdessen muss die Soziologie die variablen Prozesse und Ergebnisse von Strukturbildung in den Blick nehmen und untersuchen. Strukturbildung lässt sich besser variabel begreifen als mit dichotomen Konzepten, die ein klares Entweder-Oder voraussetzen (Fuchs 2001: 13ff).

Die vorliegende Arbeit stellt den Versuch dar, die Fruchtbarkeit der Phänomenologischen Netzwerktheorie für die Beschreibung genau solcher variabler Strukturbildung aufzuzeigen. Leider fehlt hier noch der Vergleich mit anderen Schulen, der aber mit dem skizzierten methodischen Instrumentarium insbesondere auf der Netzwerkebene möglich ist. Außer Invisible Colleges lassen sich prinzipiell viele andere soziale Phänomene wie soziale Bewegun- 
gen, politische Koalitionen oder proto-politische Systembildung, Straßengangs, Subkulturen oder soziale Milieus mit dem angebotenen Instrumentarium untersuchen. Als Gegenstand wurde hier der Theorieansatz selbst gewählt - auch um seine Autoren, deren Vernetzung, seine theoretischen Annahmen und seine empirische Anwendung im deutschen akademischen Diskurs vorzustellen.

\section{Literatur}

Abbott, Andrew (1995): Things of Boundaries, in: Social Research 62, S. 857-882.

Abbott, Andrew (1997): Of Time and Space: The Contemporary Relevance of the Chicago School, in: Social Forces 75, S. 1149-1182.

Abbott, Andrew (2001): Chaos of Disciplines, Chicago.

Alexander, Jeffrey / Bernhard Giesen / Richard Münch / Neil Smelser (Hrsg.) (1987), The Micro-Macro Link, Berkeley.

Ansell, Christopher (1997): Symbolic Networks: The Realignment of the French Working Class, 18871894, in: American Journal of Sociology 103, S. 359-390.

Baecker, Dirk (2005): Form und Formen der Kommunikation, Frankfurt/Main.

Beckert, Jens (2005): Soziologische Netzwerkanalyse, in: Dirk Kaesler (Hrsg.), Aktuelle Theorien der Soziologie, München, S. 286-312.

Borgatti, Stephen / Martin Everett (1999): Models of Core / Periphery Structures, in: Social Networks 21, S. 375-395.

Breiger, Ronald (1974): The Duality of Persons and Groups, in: Social Forces 53, S. 181-190.

Breiger, Ronald (1976): Career Attributes and Network Structure: A Blockmodel Study of a Biomedical Research Specialty, in: American Sociological Review 41, S. 117-135.

Breiger, Ronald (1995): Social Structure and the Phenomenology of Attainment, in: Annual Review of Sociology 21, S. 115-136.

Breiger, Ronald (2000): A Tool Kit for Practice Theory, in: Poetics 27, S. 91-115.

Breiger, Ronald (2004): The Analysis of Social Networks, in: Melissa Hardy / Alan Bryman (Hrsg.), Handbook of Data Analysis, London, S. 505-526.

Brint, Steven (1992): Hidden Meanings: Cultural Content and Context in Harrison White's Structural Sociology, in: Sociological Theory 10, S. 194-208.

Burt, Ronald (2004): Structural Holes and Good Ideas, in: American Journal of Sociology 110, S. 349399.

Collins, Randall (1998): The Sociology of Philosophies. A Global Theory of Intellectual Change, Cambridge-Massachusetts.

Collins, Randall (2004): Interaction Ritual Chains, Princeton.

Crane, Diana (1972): Invisible Colleges, Chicago.

DiMaggio, Paul (1992): Nadel's Paradox Revisited: Relational and Cultural Aspects of Organizational Culture, in: Nitin Nohria / Robert Eccles (Hrsg.), Networks and Organizations, Boston, S. 118-142.

DiMaggio, Paul (1997): Culture and Cognition, in: Annual Review of Sociology 23, S. $263-287$.

Emirbayer, Mustafa (1997): Manifesto for a Relational Sociology, in: American Journal of Sociology 103, S. 281-317.

Emirbayer, Mustafa / Jeff Goodwin (1994): Network Analysis, Culture, and the Problem of Agency, in: American Journal of Sociology 99, S. 1411-1154.

Emirbayer, Mustafa / Ann Mische (1998): What is Agency?, in: American Journal of Sociology 103, S. 962-1023. 
Ennis, James (1992): The Social Organization of Sociological Knowledge: Modeling the Intersection of Specialties, in: American Sociological Review 57, S. 259-265.

Erickson, Bonnie (1988): The Relational Basis of Attitudes, in: Wellman / Berkowitz 2005, S. 99-121.

Erickson, Bonnie (1996): Culture, Class, and Connections, in: American Journal of Sociology 102, S. 217-251.

Fine, Gary Alan (1995): Introduction, in: Ders. (Hrsg.): A Second Chicago School?, Chicago, S. 1-15.

Fine, Gary Alan / Sherryl Kleinman (1983): Network and Meaning: An Interactionist Approach to Structure, in: Symbolic Interaction 6, S. 97-110.

Freeman, Linton (2004): The Development of Social Network Analysis, Vancouver.

Fuchs, Stephan (2001): Against Essentialism. A Theory of Culture and Society, Cambridge-Massachusetts.

Gibson, David (2005): Taking Turns and Talking Ties: Networks and Conversational Interaction, in: American Journal of Sociology 110, S. 1561-1597.

Gould, Roger (1995): Insurgent Identities. Class, Community, and Protest in Paris from 1848 to the Commune, Chicago.

Griffith, Belver / Nicholas Mullins (1972): Coherent Social Groups in Scientific Change, in: Science 177, S. 959-964.

Hanneman, Robert / Mark Riddle (2005): Introduction to Social Network Methods, University of California, Riverside. Verfügbar über: http://faculty.ucr.edu/ hanneman/mathematica_networks.pdf [Datum des Zugriffs: 16. Februar 2008].

Hannerz, Ulf (1992): Cultural Complexity. Studies in the Social Organization of Meaning, New York.

Hollstein, Betina / Yvonne Schütze (2004): Selbstdarstellungen in der Wissenschaft am Beispiel von Danksagungen in der Soziologie, in: Zeitschrift für Pädagogik, Beiheft 48, S. 153-182.

Holzer, Boris (2006): Netzwerke, Bielefeld.

Holzer, Boris (2008): Netzwerke und Systeme: Zum Verhältnis von Vernetzung und Differenzierung, in: Christian Stegbauer (Hrsg.), Ein neues Paradigma in den Sozialwissenschaften: Netzwerkanalyse und Netzwerktheorie, Wiesbaden (im Druck).

Ikegami, Eiko (2000): A Sociological Theory of Publics: Identity and Culture as Emergent Properties in Networks, in: Social Research 67, S. 989-1029.

Knox, Hannah / Mike Savage / Penny Harvey (2006): Social Networks and the Study of Relations: Networks as Method, Metaphor amd Form, in: Economy and Society 35, S. 113-140.

Kuhn, Thomas (1970): The Structure of Scientific Revolutions, 2. Aufl., Chicago.

Leifer, Eric (1991): Actors as Observers. A Theory of Skill in Social Relationships, New York.

Lizardo, Omar (2006): How Cultural Tastes Shape Personal Networks, in: American Sociological Review 71, S. 778-807.

Martin, John Levi (2002): Power, Authority, and the Constraint of Belief Systems, in: American Journal of Sociology 107, S. 861-904.

Martin, John Levi (2003): What Is Field Theory?, in: American Journal of Sociology 109, S. 1-49.

McLean, Paul (1998): A Frame Analysis of Favor Seeking in the Renaissance: Agency, Networks, and Political Culture, in: American Journal of Sociology 104, S. 51-91.

Mische, Ann (2003): Cross-talk in Movements: Reconceiving the Culture-Network Link, in: Mario Diani / Doug McAdam (Hrsg.), Social Movements and Networks, Oxford, S. 258-280.

Mische, Ann / Philippa Pattison (2000): Composing a Civic Arena: Publics, Projects, and Social Settings, in: Poetics 27, S. 163-194.

Mische, Ann / Harrison White (1998): Between Conversation and Situation: Public Switching Dynamics across Network Domains, in: Social Research 65, S. 695-724.

Mohr, John (1994): Soldiers, Mothers, Tramps and Others: Discourse Roles in the 1907 New York City Charity Directory, in: Poetics 22, S. 327-357. 
Mohr, John (1998): Measuring Meaning Structures, in: Annual Review of Sociology 24, S. 345-370.

Mohr, John (2000): Introduction: Structures, Institutions, and Cultural Analysis, in: Poetics 27, S. 57-68.

Mullins, Nicholas (1968): The Distribution of Social and Cultural Properties in Informal Communication Networks among Biological Scientists, in: American Sociological Review 33, S. 786-797.

Mullins, Nicholas (1973): Theories and Theory Groups in Contemporary American Sociology, New York.

Mullins, Nicholas / Lowell Hargens / Pamela Hecht / Edward Kick (1977): The Group Structure of Cocitation Clusters: A Comparative Study, in: American Sociological Review 42, S. 552-562.

Padgett, John / Christopher Ansell (1993): Robust Action and the Rise of the Medici, in: American Journal of Sociology 98, S. 1259-1319.

Podolny, Joel (2001): Networks as the Pipes and Prisms of the Market, in: American Journal of Sociology 107, S. 33-60.

Scott, John (2000): Social Network Analysis. 2. Aufl., London.

Small, Henry / Belver Griffith (1974): The Structure of Scientific Literatures I: Identifying and Graphing Specialties, in: Science Studies 4, S. 17-40.

Somers, Margaret (1994): The Narrative Constitution of Identity: A Relational and Network Approach, in: Theory and Society 2, S. 605-649.

Somers, Margaret (1998): »We're No Angels«: Realism, Rational Choice, and Relationality in Social Science, in: American Journal of Sociology 104, S. 722-784.

Tilly, Charles (1998): Durable Inequality, Berkeley.

Tilly, Charles (2002): Stories, Identities, and Political Change, Lanham.

Trezzini, Bruno (1998): Theoretische Aspekte der sozialwissenschaftlichen Netzwerkanalyse, in: Schweizerische Zeitschrift für Soziologie 24, S. 511-544

Wellman, Barry (1983): Network Analysis: Some Basic Principles, in: Sociological Theory 1, S. 155-200.

Wellman, Barry / Stephen Berkowitz (Hrsg.) (1988): Social Structure. A Network Approach, Cambridge.

White, Harrison (1992): Identity and Control. A Structural Theory of Social Action, Princeton.

White, Harrison (1993): Careers and Creativity. Social Forces in the Arts, Boulder.

White, Harrison (1993b): Values Come in Styles, Which Mate to Change, in: Michael Hechter / Lynn Nadel / Richard Michad (Hrsg.), The Origin of Values, New York, S. 63-91.

White, Harrison (1995): Network Switchings and Bayesian Forks: Reconstructing the Social and Behavioral Sciences, in: Social Research 62, S. 1035-1063.

White, Harrison / Scott Boorman / Ronald Breiger (1976): Social Structure from Multiple Networks. I. Blockmodels of Roles and Positions, in: American Journal of Sociology 81, S. 730-780.

Dr. Jan Arendt Fuhse Paul F. Lazarsfeld Center for the Social Sciences

Columbia University

420 West 118th Street, Mail Code 3355

New York, NY 10027, USA

jan@fuhse.net 